\title{
Construction and Validation of Educational Material for Children with Hydrocephalus and Their Informal Caregivers
}

\section{Paloma de Aro J. Tavares', Pedro Tadao Hamamoto Filho ${ }^{2}$, Ana Sílvia S.B.S. Ferreira ${ }^{3}$, Marla Andréia G. Avila}

OBJECTIVE: To build and validate journal-type digital and printed educational material for informal caregivers (i.e., the parents who provide care) of children with hydrocephalus.

METHODS: This is a methodologic study conducted in the children's neurosurgery outpatient clinic of a university hospital. The content validity index with a concordance of $\geq 0.78$ was used for validation. Content was validated with the participation of 5 nurses and 3 neurosurgeons, and appearance was validated with the participation of 9 informal caregivers of children with hydrocephalus.

RESULTS: A 21-page journal was created to address hydrocephalus and its treatment. The content and semantics of this material were validated with a universal content validity index of 0.90 and 0.98 , respectively.

CONCLUSIONS: Content and appearance of the educational material for informal caregivers of children with hydrocephalus were successfully validated and considered suitable and user-friendly for health education.

\section{INTRODUCTION}

1 ongenital hydrocephalus occurs at a frequency of I:I00o live births; in developing countries, this number increases to 3.I6: 1000 live births. ${ }^{I}$ It is one of the most common neurosurgical diseases in childhood and is generally treated with a ventriculoperitoneal (VP) shunt. ${ }^{2,3}$ Although this treatment is quite effective in most cases, there is a considerable risk of dysfunction and infection, especially in the first year of use. ${ }^{4}$
After the birth of a child with hydrocephalus, the families, especially the mother, usually develop fear of the unknown and the unexpected in relation to the disease and of the need for surgery in the first hours or days of life. ${ }^{I, 5}$ In the course of their lives, these children have special health needs and require specific care at home from their informal caregivers (i.e., the family members who care for the child). This care usually includes preventing postoperative complications; tissue care to prevent pressure injury to the head; identifying the warning signs for intracranial hypertension; and other measures to improve hydration, nutrition, and comfort. $^{6}$ It is especially important that caregivers are able to monitor and detect changes in a child with shunted hydrocephalus. They need to promptly identify signs and symptoms of shunt malfunction and seek immediate medical assistance, as delay in treatment is hazardous and life threatening. Many families, however, care for their children using the popular knowledge, based on beliefs, customs, culture, and resources that are considered inconsistent with the practices of the health care system. ${ }^{6-9}$

Educational material in print or digital format is becoming increasingly popular for health education. A literature review stresses the importance of the Internet as a health education tool for informal caregivers to prevent significant morbidity and mortality owing to shunt failure. ${ }^{\text {IO }}$ According to this study, most informal caregivers ( $91.7 \%$ ) used the Internet, and $90.3 \%$ of these caregivers accessed the Internet from home. Most caregivers $(8 \mathrm{r} .9 \%)$ who used the Internet reported having looked for information on hydrocephalus. Of these caregivers, $52.7 \%$ considered the information they found on hydrocephalus very useful, whereas $2.5 \%$ rarely used the information or believed that it was not useful. Moreover, $89.8 \%$ of the caregivers who used the Internet reported that they would like their neurosurgeons to recommend the best resources. ${ }^{\text {Io }}$ Furthermore, the informal caregivers who used the Internet did not believe that the information they obtained was fully reliable; $57.8 \%$ reported that

\section{Key words \\ - Caregivers \\ - Health education \\ - Hydrocephalus \\ - Perioperative nursing \\ - Rehabilitation}

Abbreviations and Acronyms

CVI: Content validity index

VP: Ventriculoperitoneal
From the ${ }^{\mathbf{1}}$ Department of Nursing, ${ }^{2}$ Department of Neurology, Psychology and Psychiatry, and ${ }^{3}$ Center of Distance Education and Information Technology in Healthcare, Botucatu Medical School, UNESP-Universidade Estadual Paulista, Botucatu, São Paulo, Brazil

To whom correspondence should be addressed: Pedro Tadao Hamamoto Filho, M.D., M.Sc. [E-mail: phamamoto@fmb.unesp.br]

Citation: World Neurosurg. (2018) 114:381-390

https://doi.org/10.1016/j.wneu.2018.03.082

Journal homepage: www.WORLDNEUROSURGERY.org

Available online: www.sciencedirect.com

1878-8750/\$ - see front matter (c) 2018 Elsevier Inc. All rights reserved. 
they sometimes trusted the information on hydrocephalus obtained from the Internet. A small proportion of these caregivers (2I.7\%) always trusted the information from the Internet. Additionally, $28.6 \%$ of the caregivers used the Internet to find support groups, whereas $34.8 \%$ used the Internet to communicate with other caregivers of children with similar conditions. ${ }^{\text {IO }}$ The Internet is a popular source of information among patients and their families, although the information is not always reliable. Consequently, educational material created by the health care team to assist the patients would facilitate communication and clarify doubts regarding the reliability of information.

The lack of guidelines from the health care team or the lack of communication for this specific population after hospital discharge increases the difficulties of caregivers. ${ }^{\text {II }}$ Health education is an important intervention strategy to prepare and provide information to the caregivers, thereby increasing the quality of life of the children. ${ }^{12}$ This education can be provided using printed educational material. This form of material can potentially ensure the shared creation of knowledge and emerges as a new health promotion proposal based on the participation of the population. Manuals, leaflets, brochures, booklets, flip charts, and guidebooks all are accessible alternatives that patients and their families can read after hospital discharge to support the verbal guidelines of the health care teams, to clarify future doubts, and to assist in decision making. ${ }^{\text {II }}$ For these resources to be used effectively, however, they must be developed and validated. ${ }^{\mathrm{I} 3}$

For the target public to use educational material and benefit from this tool, it is important to translate the knowledge. Knowledge translation is defined by the Canadian Institute of Health Research as "a dynamic and iterative process that includes synthesis, dissemination, exchange, and ethically-sound application of knowledge to improve the health, provide more effective health services and products, and strengthen the healthcare system." ${ }^{\text {I4 }}$ Considering that many children must use the VP shunt throughout their lifetime, considering that care provided by the medical and nursing staff must include health education for the families during treatment, and considering the lack of appropriate educational material for children with hydrocephalus available in Brazil, it was necessary to create a printed and digital educational material for the families of patients with hydrocephalus. The aim of this study was to develop an educational material and validate it by experts and the target population in terms of content and appearance, considering semantics and appropriateness to our cultural context.

\section{MATERIALS AND METHODS}

\section{Study Design}

This research was approved by the research ethics committee of the Botucatu Medical School, UNESP-Universidade Estadual Paulista (CAAE: 64II99I7.6.0000.54II) and complies with resolution No. 466/12, which establishes the guidelines and regulatory standards for research involving humans. A methodologic descriptive study was used for the creation and validation of educational material intended for the health education of informal caregivers of children with hydrocephalus in perioperative care for placement or change of the VP shunt. Methodologic descriptive studies are employed to investigate methods used to collect and organize data, including the development, validation, and evaluation of research tools and methods, thus increasing the rigor of investigations. ${ }^{15}$

\section{Study Period and Location}

The study period was August 2016 to October 2017. The study was conducted at the Núcleo de Educação à Distância e Tecnologias da Informação em Saúde (Center of Distance Education and Information Technology in Healthcare) of the Faculdade de Medicina de Botucatu and the Children's Neurosurgery Outpatient Clinic of the Hospital das Clínicas of the Faculdade de Medicina de Botucatu, which provides care to approximately 40 children every month. This hospital is located in the center-west region of the state of São Paulo in Brazil, and it is the referral center for up to 2 million patients in 68 cities. The human development index of the region is 0.804 , and, similar to the country, social inequality is high. Therefore, as the only public tertiary health care center, the hospital assists mainly low-income patients.

\section{Population}

The experts were professionals who met at least $\mathrm{I}$ of the following criteria: a minimum of 5 years working in pediatrics or neurosurgery (teaching, research, care, or rehabilitation); experience in health education or health communication or creating and/or assessing educational/instructional materials. The experts who did not answer the online form within a period of 30 days were excluded. Eight experts were enrolled.

The informal caregivers were selected from a convenience sample at our Children's Neurosurgery Department. They were family members who looked after children with a VP shunt and assisted at the children's neurosurgery outpatient clinic. Informal caregivers who did not define themselves as being primarily responsible for the child's care, who were paid for this care, or who could not read or write were excluded.

\section{Study Protocol}

An extensive literature review was conducted to select the scientific content and construct the educational material. We reviewed books and scientific articles published within the last Io years in Portuguese or English addressing the topic of educational materials or health education on children with hydrocephalus. The needs of the children and their caregivers identified during the nursing or medical consultations and the expertise of researchers on the topic were also considered. A team of physicians and nurses created a scientific script in a language the target public could easily understand, with minimal use of technical terms.

Subsequently, a storyboard was created containing some scenes developed by the children with the authorization of their tutors. Some images were obtained from books and websites to better illustrate the scenes. The proposal was submitted to the Center of Distance Education and Information Technology in Healthcare; advertising and communication specialists designed the educational material using the iBooks Author software (Apple Inc., Cupertino, California, USA). Once the experts finalized the educational material, some adjustments were made to the written content and illustrations, such as color and font size. The 
educational material contained 2I pages, including the cover, back cover, and the presentation of characters and authors.

When the first version of the booklet was finalized, the validation process was initiated. The validation process involves 2 approaches: content and appearance, also called semantics. ${ }^{\mathrm{I}, 17}$ Content validation consisted of a search of the professional curricula platform of Brazilian researchers (www.lattes.cnpq.br) to identify experts who met the inclusion criteria. The experts were contacted by e-mail and the educational material was submitted to each expert with an assessment form that was used in another study for the construction and validation of an educational material, ${ }^{18}$ powered by the Lime Survey platform (www.limesurvey. org). Following content validation, the necessary adjustments were made, and the material was printed for the informal caregivers to validate its appearance.

The content validation criteria used in the form were based on the following 8 domains: I) objective, 2) content, 3) language, 4) relevance, 5) illustrations, 6) layout, 7) motivation, and 8) culture. Each domain should have a concordance $\geq 0.78$ to be considered suitable. The domains that did not obtain this rating were removed or reviewed according to the recommendation of the experts. To assess each domain, the experts had to mark I of the following options with their respective ratings: "totally agree"
(5 points), "agree" (4 points), "do not agree or disagree" (3 points), "disagree" (2 points), and "strongly disagree" (I point).

The objective domain refers to the goals and outcomes that the developers hope to achieve with the educational material. The content domain refers to the knowledge provided by the educational material. The language domain refers to the language characteristics, type of writing, and understanding of the concepts covered in the educational. The relevance domain assesses the level of significance of the educational material. The illustrations domain refers to the assessment of imagery in the educational material. The layout domain refers to the presentation of the educational material and whether it encourages people to read the booklet. The motivation domain refers to the desire to read the booklet. Finally, the culture domain refers to the compliance of the educational material with the target public. Figure 1 details each one of these domains. Following the content validation, the informal caregivers validated the semantics of the material.

The informal caregivers rated the material based on the following 5 domains: I) objectives and content, 2) language, 3) illustrations, 4) structure and organization, and 5) motivation. A self-applied form was used to characterize the participants and assess the concordance of the educational material. This form was adapted from another study on the construction and validation of

\begin{tabular}{|c|c|c|c|}
\hline \multicolumn{4}{|c|}{ Assessed Domains } \\
\hline Objective & Content & Language & Relevance \\
\hline $\begin{array}{ll}\checkmark & \text { Consistency with health } \\
& \text { education needs } \\
\checkmark & \text { Usefulness } \\
\checkmark & \text { Promotion of thought } \\
\checkmark & \text { Promotion of behavior } \\
& \text { and attitude shifts }\end{array}$ & $\begin{array}{ll}\checkmark & \text { Suitability for informal } \\
& \text { caregivers } \\
\checkmark & \text { Information providing } \\
\checkmark & \text { Highlights on health } \\
& \text { education } \\
\checkmark & \text { Clear text } \\
\checkmark & \text { Scientific correction } \\
\checkmark & \text { Achievement of objective } \\
\checkmark & \text { Logical sequence }\end{array}$ & $\begin{array}{ll}\checkmark & \text { Clearness and } \\
& \text { intelligibility } \\
\checkmark & \text { Adequacy to target } \\
& \text { public } \\
\checkmark & \text { Well structured } \\
\checkmark & \text { Spelling correction } \\
\checkmark & \text { Attractive writing }\end{array}$ & $\begin{array}{ll}\checkmark & \text { Key points depicted } \\
\checkmark & \text { Potential of learning } \\
& \text { transfer } \\
\checkmark & \text { Scope } \\
\checkmark & \text { Suitability for health } \\
& \text { workers } \\
\checkmark & \text { Pertinence }\end{array}$ \\
\hline Illustrations & Layout & Motivation & Culture \\
\hline $\begin{array}{ll}\checkmark & \text { Relevance to content } \\
\checkmark & \text { Expression of needed } \\
& \text { information } \\
\checkmark & \text { Number } \\
\checkmark & \text { Characters' charisma } \\
\checkmark & \text { Sufficiency of introduced } \\
\checkmark & \text { characters } \\
\checkmark & \text { Resemblance with real } \\
& \text { life }\end{array}$ & $\begin{array}{ll}\checkmark & \text { Attractiveness } \\
\checkmark & \text { Font size } \\
\checkmark & \text { Typeface } \\
\checkmark & \text { Color contrast } \\
\checkmark & \text { Text wrapping } \\
\checkmark & \text { Number of pages }\end{array}$ & $\begin{array}{ll}\checkmark & \text { Arousal of reader's } \\
& \text { interest } \\
\checkmark & \text { Attractiveness of content } \\
\checkmark & \text { Enthusiasm for readers }\end{array}$ & $\begin{array}{ll}\checkmark & \text { Appropriateness for } \\
\text { socio-cultural level of } \\
\text { target public }\end{array}$ \\
\hline
\end{tabular}

Figure 1. Items in each domain assessed by the experts. 
educational material. ${ }^{17}$ The objective and content domains refer to the goals and desired outcomes following the application of the educational material and the availability of information. The language domain refers to the language characteristics, type of writing, and understanding of the concepts covered in the educational material. The illustrations domain refers to the linguistic characteristics, type of writing, and understanding of the concepts covered in the educational material. The structure and organization domains refer to the way the guidelines are presented and the form of the educational material. The motivation domain refers to the readers' eagerness and desire to read the material. Finally, the participants were asked to make general comments and suggestions. Following the appearance validation, the necessary adjustments were made to the final version of the educational material.

\section{Analysis of Results and Statistics}

The content and appearance validation data were tabulated in Microsoft Office Excel (Microsoft Corp., Redmond, Washington) and analyzed using the content validity index $(\mathrm{CVI}) .{ }^{\mathrm{I} 8}$ The CVI measures the proportion or percentage of experts or judges who are in agreement. This method was initially used to analyze each item individually and subsequently the instrument as a whole. It consists of a Likert-type scale with scores from I to 4 . To assess relevancy/representativeness, the answers may range from $\mathrm{I}$ to 4 ( $\mathrm{I}=$ not relevant or not representative, $2=$ item needs further review to be representative, $3=$ item requires minimal review to be representative, $4=$ item is relevant or representative). The index score is calculated by adding the concordance of all items marked with 3 or 4 by the judges. Items with a score of $\mathrm{I}$ or 2 must be reviewed or removed. Thus, the CVI has also been computed as "the number of experts giving a rating of 3 or 4 to the relevancy of each item, divided by the total number of experts." I9 In this study, CVI was calculated with the items marked with 4 and 5, corresponding to the answers "agree" and "totally agree."

As there is no consensus on the ideal number of participants, a convenience sample was used, including all the selected professionals and caregivers and considering the time of data collection. It was suggested that the number of experts must be considered in the assessment of individual items. When there are $\leq 5$ experts, they must all agree for the item to be considered representative. In the case of $\geq 6$ total answers, a minimum rate of 0.7822 was recommended. This rate was applied in this study.

\section{RESULTS}

The educational material (paper and electronic formats) consisted of a journal shared between a child with hydrocephalus and her mother. In the journal, the child and mother write about their experience of VP shunt dysfunction through a logical series of events covering the following topics: definition of hydrocephalus, recognition of signs and symptoms of VP shunt dysfunction, an instructional explanation of VP shunt obstruction, and care in the perioperative period. In addition, the material consistently addresses the importance of the informal caregiver, the surgical team (nurses, neurosurgeon, and anesthesiologist), the surgical environment, and social inclusion. At the end of the material, interaction between the caregivers and the material is encouraged by providing a space to write down consultations, surgeries, questions, and reports of their own day-to-day occurrences (Figure 2).

The content was validated by 8 health care workers (physicians and nurses), and the appearance was validated by 9 informal caregivers of children with hydrocephalus. Of the 8 experts, 5 were nurses $(2$ specializing in pediatric neurosurgical care, 2 in health education, and I in rehabilitation), and 3 were physicians who specialized in pediatric neurosurgery. The average number of years of experience of the experts with hydrocephalus was 20 years (range, I4-33 years). Of the 8 experts, 4 had doctoral degrees, 2 had postdoctoral degrees, I had a master's degree, and I had a specialization. The content of the material was validated using the 8 domains, objective, content, language, relevancy, illustrations, layout, motivation, and culture, with a universal CVI of o.9o. The CVIs of the domains were $0.97,0.86,0.93,0.85,0.92,0.88,0.88$, and 0.88 (Table 1). Of all the content validation domains, $\mathrm{I} 6$ items obtained the maximum concordance of $\mathrm{I}$, whereas $\mathrm{I} 3$ items, 6 items, I item, and I item had concordances of $0.88,0.75,0.63$, and 0.05 .

Some contradictions were identified in the comments and suggestions of the experts for items related to whether the content was sufficient to reach the goals of the educational material and whether the number of pages was appropriate (CVI of 0.5 and o.63, respectively). In these 2 items, some experts thought that the content was excessive, whereas others questioned the lack of content and the lack of other clinical cases, such as the presence of neurologic sequelae and the first placement of the VP shunt.

In the language domain, for the item referring to the use of attractive writing, the CVI was 0.75 , and the experts recommended changing the terms "device" and "blocked" to "tube" and "clogged." These terms were changed and later validated by the informal caregivers.

In the relevance domain, the item relating to the inclusion of necessary topics in the booklet for the informal caregivers of children with hydrocephalus had a CVI of 0.75 , and the experts recommended the addition of the team on duty, as the neurosurgeon is not always present, and of other clinical cases. A team on duty was added to the text, and it was mentioned in page 16 of the educational material that the nursing ward has children of other age groups with hydrocephalus and children with other neurologic deficits. In the item referring to whether the booklet is suitable for any health care worker, the experts indicated that although the educational material was simple for training health care professionals, the objective was for health care workers to use the booklet to educate the informal caregivers of children with hydrocephalus (CVI of 0.75 ).

In the domain of illustration, for the item referring to the sufficient number of characters and situations (CVI of 0.75 ), the experts recommended adding a short introduction of the character Breno, the brother of the main character, as he is mentioned in the story. This introduction was added.

Concerning the layout, a contradiction was detected regarding the readability of the selected font size and the appropriate size and font of the wording (CVI of 0.75 and 0.88 , respectively).

In the motivation domain, the item referring to the title being attractive enough to attract readers had a concordance of 0.75 , and the experts suggested changing the title from "Laura's Diary: Overcoming Hydrocephalus and Its Treatment" to "Laura's Diary: Understanding Hydrocephalus and Its Treatment." 


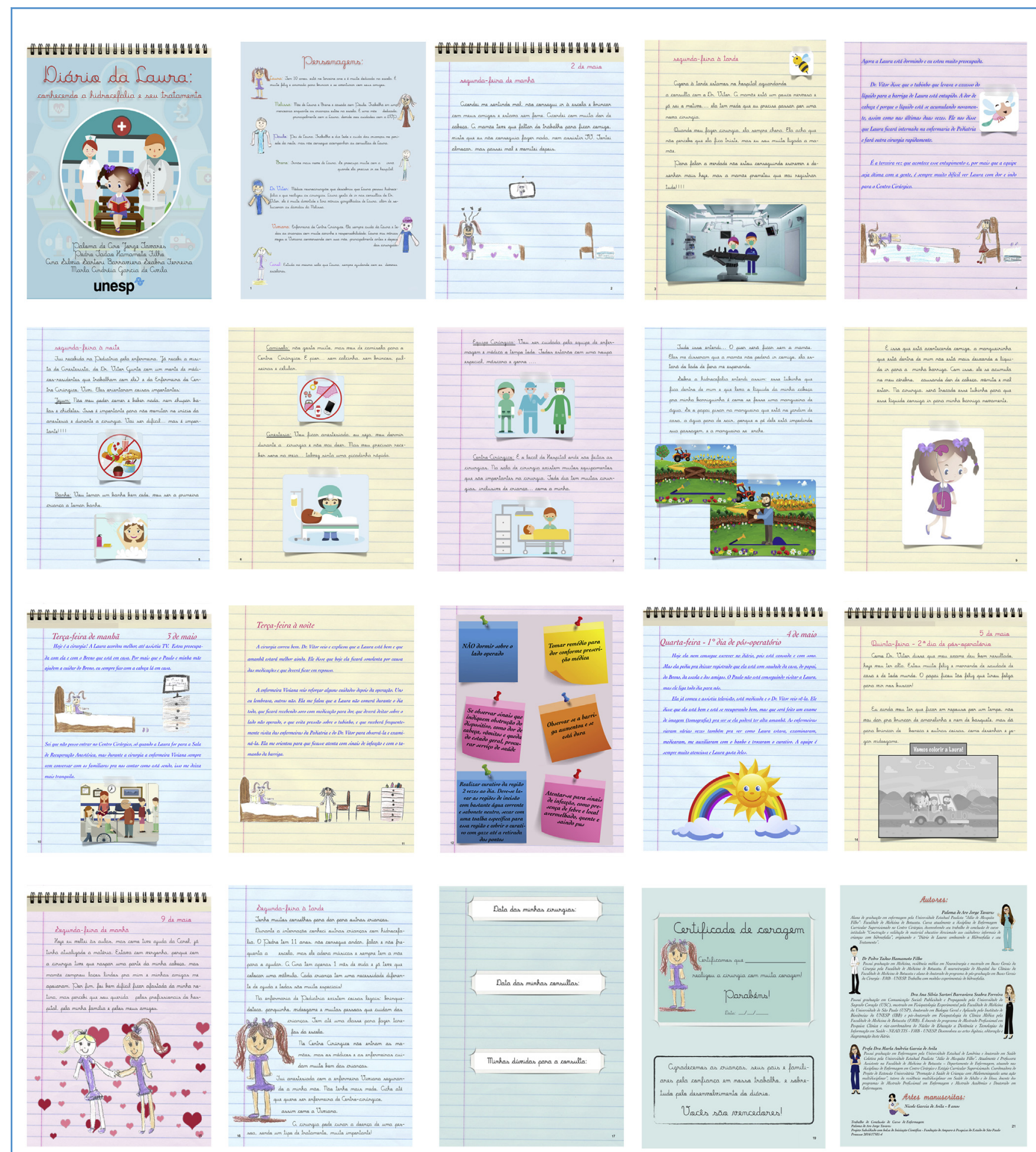

Figure 2. Images of the educational material, "Laura's Diary: Understanding Hydrocephalus and Its Treatment" (Botucatu, São Paulo, Brazil; 2017).

Regarding the relevance of the topics covered, all were categorized as "highly relevant" or "relevant." After validation by the experts, the material was redesigned before it was validated by the caregivers.
Nine caregivers participated in the appearance validation. All caregivers were mothers of the children, with an average age of 27.2 (range, $19-36$ years). Level of education of informal 
Table 1. Content Validity Index According to the Experts Regarding Objective, Content, Language, Relevance, Illustrations, Layout, Motivation, and Culture, in Absolute Frequency and Average Concordance Rate

$$
\text { Scores }(\boldsymbol{N}=8)
$$

\section{Evaluated Items}

1 Objective

1.1 Consistency with health education needs

1.2 Usefulness for health education

1.3 Promotion of thought on perioperative care

1.4 Promotion of behavior and attitude shifts

Subtotal

Concordance rate

2 Content

\begin{tabular}{|l|l|l|}
\hline 2.1 Suitability for informal caregivers & 3 & 5 \\
\hline 2.2 Information providing & 2 & 6 \\
\hline 2.3 Highlights on health education & 2 & 5 \\
\hline 2.4 Clearness and objectiveness of text & 2 & 5 \\
\hline 2.5 Scientific correction & 3 & 4 \\
\hline 2.6 Achievement of objective & 1 & 3 \\
\hline 2.7 Logical sequence & 2 & 5 \\
\hline Subtotal & 15 & 33 \\
\hline Concordance rate & & \\
\hline
\end{tabular}

3 Language

3.1 Clearness and intelligibility

3.2 Adequacy to the target public

3.3 Well structured

3.4 Spelling correction

3.5 Attractive writing

Subtotal

Concordance rate

\section{TA}

A

\begin{tabular}{|l|l|}
\hline 3 & 5 \\
\hline 4 & 4 \\
\hline 1 & 7 \\
\hline & 6 \\
\hline & 2 \\
\hline
\end{tabular}

NAND

D

SD

CVI

\begin{tabular}{|c|c|c|c|}
\hline 0 & 0 & 0 & 1 \\
\hline 0 & 0 & 0 & 1 \\
\hline 0 & 0 & 0 & 1 \\
\hline 1 & 0 & 0 & 0.88 \\
\hline 1 & 0 & 0 & \\
\hline
\end{tabular}

0.97

\begin{tabular}{|c|c|c|}
\hline 0 & 0 & 1 \\
\hline 0 & 0 & 1 \\
\hline 0 & 0 & 0.88 \\
\hline 0 & 0 & 0.88 \\
\hline 0 & 0 & 0.88 \\
\hline 0 & 0 & 0.5 \\
\hline 0 & 0 & 0.88 \\
\hline 0 & 0 & \\
\hline
\end{tabular}

\begin{tabular}{|l|l|}
\hline 3 & 5 \\
\hline 3 & 4 \\
\hline 5 & 3 \\
\hline 4 & 4 \\
\hline 2 & 4 \\
\hline 17 & 20 \\
\hline
\end{tabular}

4 Relevancy

4.1 Key points depicted

4.2 Potential of learning transfer

4.3 Scope

4.4 Suitability for health

4.5 Pertinence

Subtotal

Concordance rate

\begin{tabular}{l|l}
0 \\
0 \\
0 \\
0 \\
2 \\
4
\end{tabular}

0
1
0
0
0
1

\begin{tabular}{|c|c|}
\hline 0 & 1 \\
\hline 0 & 0.88 \\
\hline 0 & 1 \\
\hline 0 & 1 \\
\hline 0 & 0.75 \\
\hline 0 & \\
\hline
\end{tabular}

0.90

\begin{tabular}{|c|c|c|c|c|c|}
\hline 3 & 4 & 1 & 0 & 0 & 0.88 \\
\hline 3 & 4 & 1 & 0 & 0 & 0.88 \\
\hline 1 & 5 & 2 & 0 & 0 & 0.75 \\
\hline 1 & 5 & 1 & 1 & 0 & 0.75 \\
\hline 6 & 2 & 0 & 0 & 0 & 1 \\
\hline 14 & 20 & 5 & & 1 & 0 \\
\hline
\end{tabular}

5 Illustrations

\begin{tabular}{|c|c|c|c|c|c|c|}
\hline 5.1 Relevance to content & 5 & 3 & 0 & 0 & 0 & 1 \\
\hline 5.2 Expression of needed information & 4 & 3 & 1 & 0 & 0 & 0.88 \\
\hline 5.3 Number of illustrations & 6 & 2 & 0 & 0 & 0 & 1 \\
\hline
\end{tabular}




\section{Table 1. Continued}

\begin{tabular}{|c|c|c|c|c|c|c|}
\hline \multirow[b]{2}{*}{ Evaluated Items } & \multicolumn{5}{|c|}{ Scores $(N=8)$} & \multirow[b]{2}{*}{ CVI } \\
\hline & TA & A & NAND & D & SD & \\
\hline 5.4 Characters' charisma & 4 & 3 & 1 & 0 & 0 & 0.88 \\
\hline 5.5 Sufficiency of introduced characters & 4 & 2 & 2 & 0 & 0 & 0.75 \\
\hline 5.6 Resemblance with real life & 3 & 5 & 0 & 0 & 0 & 1 \\
\hline Subtotal & 26 & 18 & 4 & 0 & 0 & \\
\hline Concordance rate & \multicolumn{6}{|c|}{0.92} \\
\hline \multicolumn{7}{|l|}{6 Layout } \\
\hline 6.1 Attractiveness & 3 & 5 & 0 & 0 & 0 & 1 \\
\hline 6.2 Font size & 2 & 5 & 0 & 1 & 0 & 0.88 \\
\hline 6.3 Typeface & 0 & 6 & 1 & 1 & 0 & 0.75 \\
\hline 6.4 Color contrast & 4 & 4 & 0 & 0 & 0 & 1 \\
\hline 6.5 Text wrapping & 2 & 6 & 0 & 0 & 0 & 1 \\
\hline 6.6 Number of pages & 2 & 3 & 3 & 0 & 0 & 0.63 \\
\hline Subtotal & 13 & 29 & 4 & 2 & 0 & \\
\hline Concordance rate & & & 0.88 & & & \\
\hline \multicolumn{7}{|l|}{7 Motivation } \\
\hline 7.1 Arousal of readers' interest & 2 & 4 & 2 & 0 & 0 & 0.75 \\
\hline 7.2 Attractiveness of content & 3 & 5 & 0 & 0 & 0 & 1 \\
\hline 7.3 Enthusiasm for readers & 4 & 3 & 1 & 0 & 0 & 0.88 \\
\hline Subtotal & 9 & 12 & 3 & 0 & 0 & \\
\hline Concordance rate & \multicolumn{6}{|c|}{0.88} \\
\hline \multicolumn{7}{|l|}{8 Culture } \\
\hline 8.1 Appropriateness for sociocultural level of target public & 2 & 5 & 1 & 0 & 0 & 0.88 \\
\hline Subtotal & 2 & 5 & 1 & 0 & 0 & \\
\hline Concordance rate & \multicolumn{6}{|c|}{0.88} \\
\hline
\end{tabular}

caregivers was <IO years for 2 caregivers, IO-I3 years for 6 caregivers, and $>$ I3 years for $\mathrm{I}$ caregiver. Five of the caregivers were married, and 4 were single. Regarding current occupation, 7 caregivers were unemployed, I worked as a production assistant, and I worked as a house cleaner. The average age of the children of the informal caregivers was 2.46 years (range, 55 days to 7 years). Five children were boys. Of the 9 children, 7 did not attend kindergarten or school. The average number of VP shunt revisions was 6 procedures (range, $\mathrm{I}-8$ ).

The appearance validation was divided into 4 domains, objective and content, language, illustrations, and organization and motivation, all with a maximum concordance of I except language, with CVI of 0.92. The universal CVI was 0.98 .

In the language domain, the item pertaining to the size of the fonts had a CVI of 0.67 because the caregivers reported difficulties in understanding the writing of the child with hydrocephalus in terms of font size and type. Consequently, the font type was changed, and the size was increased. The caregivers also suggested changing the term "ventriculoperitoneal shunt" to "VP shunt," as this term is more common among the families and the acronym is easier to say.

Three informal caregivers stressed the importance of the booklet to support the target population:

I. "I had never seen educational material for children with hydrocephalus. We find things on the Internet that are not always true and reliable."

2. "If I had read this here before, it would have been much easier to deal with the situation and care. There is a shortage of resources like this for the parents of children with hydrocephalus."

3. "I loved it. I'm going to show it to the other mothers of an online group I joined." 


\section{DISCUSSION}

The material proposed in this study was created as selfexplanatory material for health care workers to use in the health education of families who care for children with VP shunts, who generally undergo various surgeries in their lifetime. ${ }^{20,2 I}$ These children and their families are faced with a lot of stress when they are informed that surgery is required and when they are referred to the surgical unit. Lack of knowledge about the surgical environment, the surgery itself, and the child's separation from the family are widely discussed as stress factors in the literature. ${ }^{22,23}$ Therefore, providing information on the perioperative period and including the informal caregiver in the care are important coping strategies. ${ }^{24}$ Accordingly, our educational material can meet some of these needs. Similarly, humanized care, communication, and the presence of confident and suitable professionals for the procedure bring relief and tranquility to the patients and help build a relationship of trust.

As also found in the literature, educational material can be read at home, and the information can be shared with friends and other family members. From several studies that reported on online support intended for children and adolescents with chronic disease, it was found that families believed that the online support tools met their needs for information, supported home care, helped manage the care of children and adolescents with chronic illness, ${ }^{25,26}$ and often provided emotional support. ${ }^{27,28}$ Online information is considered a complement to the guidelines provided by health care workers because such information help the caregivers understand the medical terms and clarify information on the diagnosis and care of the child. ${ }^{29}$ The health care workers reported the importance of checking the quality of the information in these tools and of updating the information to ensure that it is useful and appropriate for decision making. ${ }^{30}$ Moreover, they suggested the use of content with digital resources, such as videos and cartoons. ${ }^{10,30}$ These data reinforce the importance of the online availability of the proposed material, as well as the assessment made by the experts to guarantee the content quality.

Experts from different areas were selected for validation to ensure differences in opinion and approach to the same topic, as recommended in the literature ${ }^{3 \mathrm{I}}$ and other studies that used this method. ${ }^{32,33}$ The experience of the experts was essential for the validation of the content of the educational material and for enhancing the work, especially with the changes of the title and some terms as well as the addition of the team on duty.

The characterization of the informal caregivers in this study revealed a predominance of women and mothers as the primary caregivers, which is confirmed in other studies. ${ }^{\mathrm{I}, 5,6,34}$ The mothers generally care for the child, regardless of age, even if they work outside the home. At the hospital, the mothers usually accompany the child. Some studies claimed that the mothers believe that no other person is more capable of protecting and taking care of their children. ${ }^{35}$

By occupation, $77.8 \%$ of the informal caregivers were unemployed and were fully dedicated to caring for their children with hydrocephalus, as similarly observed in other Brazilian studies. ${ }^{36,37}$ As children with chronic disease need their mother's care, these women often quit their jobs and assume the exclusive role of caregivers. Consequently, the caregivers are often exposed to emotional exhaustion. ${ }^{5}$ The families consider health care workers the primary source of reliable information, which reinforces the importance of their involvement in the development of information provided in booklets, on websites, and in online support programs. ${ }^{29,30,38}$ The family members also value the quality of online information ${ }^{29,39}$ and the possibility of exchanging experiences between users. ${ }^{40,4 \mathrm{I}}$ To increase the credibility of the information, adapt the content, and develop tools to meet the needs and desires of the users of online support services, it is crucial to include trained local professionals who are familiar with the context of these families and their children and/or adolescents with chronic illnesses. ${ }^{4-43}$

Well-written materials with easy-to-understand information improves knowledge and reader satisfaction, develops skills, and facilitates autonomy and adherence, enabling readers to understand how their own actions affect their standard of health and facilitating decision making. For health-related messages to be effectively communicated, they must be well planned, precise, relevant, and well understood. The vocabulary must be coherent to the target public, and it must be attractive and easy to read and understand..$^{\text {I }}$ In our educational material, language was the lowest rated domain by the experts. The CVI of this domain was below the minimum range of the method owing to the use of some technical terms and difficulties in reading Laura's entries because of the chosen font size and type. The informal caregivers also contributed to this stage by informing that even after the color and font of the mother's and child's writing were changed, as suggested by the experts, they still had difficulties reading the text. Therefore, the adjustments of the educational material were pivotal to make it attractive and easy to understand.

The illustrations created by the graphic designers were well rated by the experts and the informal caregivers. They were considered relevant, easy to understand, coherent with the text, and quantitatively and qualitatively appropriate. According to the literature, simple language and/or the use of imagery can reduce communication barriers and increase the efficiency and coverage of information. . $^{3 \mathrm{I}}$

Health education can ensure that the families are included in the care plan of the patients by valuing and sharing of knowledge and skills, which boosts their confidence and empowers them to assume their role as caregivers; this takes into consideration that health care workers do not always recognize the suffering of the caregivers or their need to be technically and emotionally prepared to provide this level of care. ${ }^{33}$ The health care workers are responsible for ensuring that this information is provided and that the patients and their caregivers receive effective health education. A literature review that assessed the production and/or use of printed materials in health education suggested that communication and educational materials can also open new paths to health promotion through the participation of the population in the construction of knowledge or through other means implemented in the future. ${ }^{\text {Io }}$ As reported by some authors, the recipients of educational materials play an active role in the production of meaning; therefore, in addition to the quality of the material, it is necessary to investigate how effectively the reception and mediation processes occur. ${ }^{\text {IO }}$ 
Based on the contribution and reports of the evaluators, the educational material on hydrocephalus presented here was prepared appropriately, and the evaluators considered it innovative in the field of health care. This educational material included the family in the process of care and can serve as a resource to minimize the burden of caregivers. ${ }^{30}$ Furthermore, the informal caregivers and the experts clearly recognized the importance of this topic and mentioned a lack of information, studies, and means to divulge information on hydrocephalus, its treatment, and perioperative therapy. The use of printed material facilitates the process of acquiring, exploiting, and expanding knowledge, based on 3 functions: reinforcing information and oral discussions, serving as a guide to clarify future doubts, and supporting decision making. ${ }^{{ }^{\mathrm{I}}}$

Some hospitals, organizations, and shunt companies have their own educational materials for patients with hydrocephalus and their families, such as the materials from the Hydrocephalus Association (www.hydroassoc.org). However, these materials were not fully appropriate to our cultural context, and most of them are not available in Portuguese. Therefore, development and validation of our educational material were imperative. Furthermore, we added innovation by composing the booklet through the perspective of the child and the caregiver dealing with shunt malfunction.

Finally, our educational material is available free of charge in printed and digital format. However, only after the material has been implemented and clinically validated will it be possible to verify its acceptance by the families and whether the material contributed to health education. The digital format is available for
iOS devices in the Apple Store and on the institution's web page for free download.

A limitation of this study is the use of a single center to validate the semantics and the nonclinical application of the educational material. Socioeconomic and educational levels of the informal caregivers may have influenced the results. Therefore, our data should be applied with caution to different social contexts. The next steps of this study must address these concerns. An adapted material with a boy as the main character can also be developed. Finally, it will be important to validate the effectiveness of the material in teaching families the concepts they need to know for better caregiving.

\section{CONCLUSIONS}

Communication is a tool nurses and physicians use in care. Written communication is a widely used method of providing health education information. Consequently, free educational material was created in the form of a 2I-page journal entitled, "Laura's Diary: Understanding Hydrocephalus and Its Treatment," to help recognize the role of nurses, minimize the negative impact of surgery, and empower families. The educational material presented in this study enables the provision of humanized care, strengthens the link between the health care professionals and the families, encourages the participation of informal caregivers, and contributes to patient recovery. Printed and digital educational materials do not replace verbal guidelines; however, they can facilitate learning by allowing home access, increasing understanding, and encouraging the sharing of information between family members and friends.

\section{REFERENCES}

I. Melo JR, Vieira KA, Miranda T. Stress in caregivers of children with hydrocephalus. Rev Bras Neurol Psiquiatr. 20I4;18:3-I2.

2. Toma AK, Tarnaris A, Kitchen ND, Watkins LD. Ventriculosinus shunt. Neurosurg Rev. 2010;33: I47-I52.

3. Rocco FM, Saito ET, Fernandes AC. Profile of the patients with myelominingocele from the Associação de Assistência à Criança Deficiente (AACD) in São Paulo-SP, Brasil. Acta Fisiatr. 2007;14: I3O-I33.

4. Chakraborty A, Drake JM, Warf BC. Methods for cerebrospinal fluid diversion in pediatric hydrocephalus: from scope to shunt. In: QuiñonesHinojosa A, ed. Schmidek \& Sweet's Operative Neurosurgical Techniques: Indications, Methods, and Results. 6th ed. Philadelphia, PA: Saunders; 2012: $63 \mathrm{I}-653$.

5. Figueiredo SV, Sousa AC, Gomes IL. Children with special health needs and family: implications for nursing. Rev Bras Enferm. 2016;69:88-95.

6. Oliveira DMP, Pereira CU, Freitas ZMP. Nursing care to the child with hydrocephalus: an integrative literature review. In: Pereira CU, ed. Neurocirurgia Pediátrica. Rio de Janeiro: Revinter; 2000: $57-60$.
7. Carneiro TM. Living the caring and healing experiences as a family member in a hospital. Rev Bras Enferm. 2008;6r:390-394.

8. Collet N, Rocha SM. Hospitalized child: care sharing between mother and nursing. Rev LatinoAm Enfermagem. 2004;I2:I9I-I97.

9. Gomes GC, Oliveira PK. Family experience in the hospital during child hospitalization. Rev Gaúcha Enferm. 2012;33:165-171.

io. Naftel RB, Safiano NA, Falola MI, Shannon CN, Wellons JC, Johnston JM. Technology preferences among caregivers of children with hydrocephalus. J Neurosurg Pediatr. 2013;II:26-36.

II. Freitas FV, Rezende Filho LA. Communication models and use of printed materials in healthcare education: a bibliographic survey. Comunic Saude Educ. 20II;I5:243-256.

I2. Neves ET, Cabral IE. Caring of children with special health care needs: challenges to families and pediatric nursing. Rev Eletr Enf. 2009;II: 527-538.

13. Teixeira E. Technologies in nursing: productions and trends for health education with the community. Rev Eletr Enf. 2010;12:598.

14. Tetroe J. Knowledge translation at the Canadian Institutes of Health Research: a primer. Focus Technical Brief. 2007;18:I-8.
15. Lobiondo-Wood G, Haber J. Nursing Research Methods and Critical Appraisal for Evidence Based Practice. 5th ed. Rio de Janeiro: Guanabara Koogan; 2005 .

I6. Pasquali L. Psicometria: Test Theory in Psychology and Education. 2nd ed. Petrópolis: Vozes; 2003.

17. Clarck-Carter D. Quantitative Research in Psychology: From Experimental Design to Research Report. Benito Juarez, Mexico: Oxford University Press; 2002.

I8. Maniva SJCF. Elaboration and validation of educational technology on stroke for prevention of recurrence. Thesis. Faculdade de Farmácia, Odontologia e Enfermagem, Universidade Federal do Ceará, Fortaleza, 2016.

I9. Alexandre NMC, Coluci MZO. Content validity in the development and adaptation processes of measurement instruments. Ciênc Saúde Coletiva. 20II;16:306I-3068.

20. Alcântara MCM, Silva FAA, Castro ME, Moreira TMM. Clinical characteristics of children using ventricular derivations for hydrocephalus treatment. Rev Rene Fortaleza. 20II;12:776-782.

2I. Gupta N, Park JB, Kranz DA, Wrensch M, Wu IW. Long-term outcomes in patients with treated childhood hydrocephalus. J Neurosurg. 2007;106: 334-339.

22. Sampaio CEP, Ventura DSO, Batista IF Antunes TSC. Companions' of children who undergo surgical procedures and their feelings: 
experiences perioperative period. Rev Min Enferm. 2009;13:558-564.

23. Pyló RM, Peixoto MG, Bueno KMP. The caregiver in the child/adolescent hospitalization context. Cad Ter Ocup UFSCar São Carlos. 2015;23:855-862.

24. Pires MPO, Pedreira MLG, Peterlini MAS. Surgical Safety In Pediatrics: practical application of the Pediatric Surgical Safety Checklist. Rev Lat Am Enfermagem. 2015;23:II05-III2.

25. Al-Jasmi F, Moldovan I, Clarke JTR. Hunter disease eClinic: interactive, computer-assisted, problem-based approach to independent learning about a rare genetic disease. BMC Med Educ. 2010;10:72.

26. De Rouck S, Leys M. Illness trajectory and Internet as a health information and communication channel used by parents of infants admitted to a neonatal intensive care unit. J Adv Nurs. 2013;69:I489-I499.

27. Coulson NS, Greenwood N. Families affected by childhood cancer: an analysis of the provision of social support within online support groups. Child Care Health Dev. 20I2;38:870-877.

28. Patterson P, McDonald FE, Orchard P. A new Australian online and phone mental health support service for young people living with cancer. Australas Psychiatry. 2014;22:165-169.

29. Nordfeldt S, Ängarne-Lindberg T, Nordwall M, Krevers B. Parents of adolescents with type I diabetes-their views on information and communication needs and internet use. A qualitative study. PLoS One. 2013;8:e62096.

30. Swallow VM, Hall AG, Carolan I, Santacroce S, Webb NJ, Smith T. Designing a web-application to support home-based care of childhood ckd stages 3-5: qualitative study of family and professional preferences. BMC Nephrol. 20I4;15:34.
3I. Nietsche EA, Teixeira E, Medeiros HP. Assistiveeducational technologies: a possibility for the empowerment of the nurse Porto Alegre: Moriá. Rev Rene. 2014;15:I85-I86.

32. Teles LMR, Oliveira AS, Campos FC, Lima TM, Costa CC, Gomes LFS, et al. Development and validating an educational booklet for childbirth companions. Rev Esc Enferm USP. 2014;48:977-984.

33. Benevides JL, Coutinho JFV, Pascoal LC, Joventino ES, Martins MC, Gubert FA, et al. Development and validation of educational technology for venous ulcer care. Rev Esc Enferm USP. 2016;50:309-316.

34. Macedo EC, Silva LR, Paiva MS, Ramos MNP. Burden and quality of life of mothers of children and adolescents with chronic illnesses: an integrative review. Rev Lat Am Enfermagem. 2015;23: 769-777.

35. Deeken JF, Taylor KL, Mangan P, Yabroff KR, Ingham JM. Care for the caregivers: a review of self-report instruments developed to measure the burden, needs, and quality of life of informal caregivers. J Pain Symptom Manage. 2003;26: 922-953.

36. Cavalari KN, Hamamoto Filho PT, Caldeira SM, de Carvalho Nunes HR, Lima FM, de Avila MA. Functional independence of children with myelomeningocele: is it associated with the informal caregivers' burden? J Pediatr Nurs. 2017;36:232-235.

37. Moreira MD, Nóbrega MML, Silva MIT. Written communication: contribution for the elaboration of educational material in health. Rev Bras Enferm. 2003;56:184-I88.

38. Swallow V, Carolan I, Smith T, Webb NJA Knafl K, Santacroce S, et al. A novel interactive health communication application (IHCA) for parents of children with long-term conditions: development, implementation and feasibility assessment. Inform Health Soc Care. 20I6;4I:20-46.
39. Carolan I, Smith T, Hall A, Swallow VM Emerging communities of child-healthcare practice in the management of long-term conditions such as chronic kidney disease: qualitative study of parents' accounts. BMC Health Serv Res. 20I4;I4: 292

40. Swallow VM, Knafl K, Santacroce S, Campbell M, Hall AG, Smith T, et al. An interactive health communication application for supporting parents managing childhood long-term conditions: outcomes of a randomized controlled feasibility trial. JMIR Res Protoc. 20I4;3:e69.

4I. Sterling L, Nyhof-Young J, Blanchette VS Breakey VR. Exploring internet needs and use among adolescents with haemophilia: a website development project. Haemophilia. 20I2;I8:2I6-22I.

42. Byczkowski TL, Munafo JK, Britto MT. Family perceptions of the usability and value of chronic disease web-based patient portals. Health Informatics J. 20I4;20:I5I-I62.

43. Mazza VA, Lemos DS, Guimarães SL, Ferreira de Lima V, Felix JV. Online support for chronically ill children and adolescents: an integrative review. Av Enferm. 2017;35:63-76.

Conflict of interest statement: This work was supported by FAPESP_Fundação de Amparo à Pesquisa do Estado de São Paulo (grant number 16/17581-4).

Received 2 January 2018; accepted 10 March 2018

Citation: World Neurosurg. (2018) 114:381-390. https://doi.org/10.1016/j.wneu.2018.03.082

Journal homepage: www.WORLDNEUROSURGERY.org Available online: www.sciencedirect.com 1878-8750/\$ - see front matter (C) 2018 Elsevier Inc. All rights reserved. 